\section{ORIGINAL RESEARCH}

\author{
J.-W. Jeong \\ A.K. Kumar
}

S.K. Sundaram

H.T. Chugani

D.C. Chugani

\title{
Sharp Curvature of Frontal Lobe White Matter Pathways in Children with Autism Spectrum Disorders: Tract-Based Morphometry Analysis
}

\begin{abstract}
BACKGROUND AND PURPOSE: Because we had previously observed geometric changes of frontal lobe association pathways in children with ASD, in the present study we analyzed the curvature of these white matter pathways by using an objective TBM analysis.
\end{abstract}

MATERIALS AND METHODS: Diffusion tensor imaging was performed in 32 children with ASD and 14 children with typical development. Curvature, FA, AD, and RD of bilateral AF, UF, and gCC were investigated by using the TBM group analysis assessed by $\mathrm{P}_{\mathrm{FDR}}$ for multiple comparisons.

\begin{abstract}
RESULTS: Significantly higher curvatures were found in children with ASD, especially at the parietotemporal junction for $\mathrm{AF}$ (left, $\mathrm{P}_{\mathrm{FDR}}<.001$; right, $\mathrm{P}_{\mathrm{FDR}}<.01$ ), at the frontotemporal junction for UF (left, $\mathrm{P}_{\mathrm{FDR}}<.005$; right, $\left.\mathrm{P}_{\mathrm{FDR}}<.03\right)$, and at the midline of the $\mathrm{gCC}\left(\mathrm{P}_{\mathrm{FDR}}<.0001\right)$. RD was significantly higher in children with $A S D$ at the same bending regions of $A F$ (left, $P_{F D R}<.03$, right, $P_{F D R}<.02$ ), UF (left, $\left.\mathrm{P}_{\mathrm{FDR}}<.04\right)$, and $\mathrm{gCC}\left(\mathrm{P}_{\mathrm{FDR}}<.01\right)$.
\end{abstract}

CoNCLUSIONS: Higher curvature and curvature-dependent RD changes in children with ASD may be the result of higher attenuation of thinner axons in these frontal lobe tracts.

\begin{abstract}
ABBREVIATIONS: $A D=$ axial diffusivity; $A F=$ arcuate fasciculus; $A S D=$ autism spectrum disorders; $\mathrm{CC}=$ corpus callosum; $\mathrm{Cg}=$ cingulum; $\mathrm{DTI}=$ diffusion tensor imaging; $\mathrm{FA}=$ fractional anisotropy; $\mathrm{gCC}=$ genu of corpus callosum; $\mathrm{GM}=$ gray matter; $\mathrm{MD}=$ mean diffusivity; $\mathrm{MNI}=$ Montreal Neurologic Institute; $P_{\mathrm{FDR}}=$ false discovery rate $P$ value; $\mathrm{RD}=$ radial diffusivity; $\mathrm{ROI}=$ region of interest; TBM = tract-based morphometry; TD = typically developing; UF = uncinate fasciculus
\end{abstract}

A SD are neurodevelopmental disorders behaviorally characterized by impaired language and reciprocal social interaction, accompanied by repetitive and stereotypical behaviors. Although conventional neuroimaging in patients with ASD has failed to identify specific abnormalities, several MR DTI studies ${ }^{1-7}$ have reported abnormalities of white matter structure, including changes in FA, MD, AD, and RD. Significant changes in these parameters were reported in several major white matter tracts, such as the gCC, AF, UF, internal capsule, external capsule, forceps minor, and corticospinal tract.

In addition to changes in diffusion parameters, potential changes in tract geometry (length, attenuation, and volume) were suggested previously based upon conventional tractography methods from our group. ${ }^{6}$ Children with ASD showed shorter length of left UF; increased length, volume, and attenuation of right UF; increased length and attenuation of CC; and higher attenuation of left $\mathrm{Cg}$ compared with TD children. ${ }^{6}$ Furthermore, the display of conventional streamline tractography ${ }^{8}$ showed that the right UF in children with ASD seemed to course beyond the temporal lobe and then turned sharply into the temporal cortex. Based on this observation, we pre-

Received December 9, 2010; accepted after revision January 17, 2011.

From Carman and Ann Adams Departments of Pediatrics (J.-W.J., A.K., S.K.S., H.T.C., D.C.C.), Neurology (J.-W.J., A.K., S.K.S., H.T.C.), and Radiology (H.T.C., D.C.C.), Wayne State University School of Medicine, and Translational Imaging Laboratory (J.-W.J., A.K., S.K.S., H.T.C., D.C.C.), Children's Hospital of Michigan, Detroit, Michigan.

Please address correspondence to Jeong-Won Jeong, PhD, Departments of Pediatrics and Neurology, Wayne State University, PET Center, Children's Hospital of Michigan, 3901 Beaubien Blvd, Detroit, MI 48201; e-mail: jeongwon@pet.wayne.edu

http://dx.doi.org/10.3174/ajnr.A2557 sumed that objective investigation of the geometry and morphometry of these fiber pathways might provide additional insight into the neuronal organization and morphology associated with ASD.

Here, we used TBM analysis to differentiate alterations in the curvature (ie, first eigenvector change per unit length) of major frontal lobe tracts in children with ASD. The TBM anal$\mathrm{ysis}^{9}$ was originally proposed to investigate subtle alterations in diffusivity parameters of bilateral $\mathrm{AF}$ and $\mathrm{Cg}$ in normal adults. This method spatially normalizes the fibers of multiple subjects in standard space and then registers the coordinates of individual fibers to those of a group standard fiber, followed by statistical analysis of diffusivity parameters only at the common coordinates of group subjects.

Unlike other DTI analyses such as tract-based spatial statistics ${ }^{10}$ and tissue-specific smoothing-compensated voxelbased analysis ${ }^{11}$ that use diffusion map (eg, FA or b0 image) for voxel-wise intersubject registration, the TBM analysis uses native white matter trajectory to register spatial coordinates of an individual subject's tracts to a common coordinate system that represents a given population. Thus, TBM provides a feasible way to evaluate the curvature of individual tracts at any point of their course in $3 \mathrm{D}$ space. Also, it may result in lower error rates in registering DTI parameters across subjects, which highly depends on the differences of individual white matter tract morphology. Furthermore, statistical analysis of DTI parameters at the common coordinate system may enhance the reliability of group comparison because the parameter values are directly sampled at anatomically equivalent tract points.

Here, we analyzed the curvature of the major frontal white 
matter pathways, including bilateral AF, UF, and gCC, by using an objective TBM analysis, in children with ASD. We also evaluated how the changes in curvature of these tracts relate to various diffusion parameters, such as $\mathrm{FA}, \mathrm{AD}$, and $\mathrm{RD}$.

\section{Materials and Methods}

\section{Subjects}

Thirty-two children with a diagnosis of ASD (mean age, $58.8 \pm 22.64$ months; 29 males) and 14 TD children (mean age, $67.36 \pm 23.81$ months; 11 males; data from this group of children were reported in Kumar et al 2010 ${ }^{6}$ ) underwent MR imaging with DTI and developmental-behavioral assessments. All the patients had been referred to the Children's Hospital of Michigan Neurology Clinic. The inclusion criteria for the ASD group included the following: 1) a diagnosis of autistic disorder, Asperger disorder, or pervasive developmental disorder not otherwise specified made by pediatric neurologists by using the Diagnostic and Statistical Manual of Mental Disorders, 4th edition, text revision criteria; 2) scores $>15$ on the Social Communication Questionnaire $^{12}$; and 3) scores $>85$ on the autism quotient of the Gilliam Autism Rating Scales. ${ }^{13}$

We did not attempt to distinguish between different diagnostic categories within the ASD group, because current data do not support the notion that strictly behaviorally defined autism is a homogeneous disorder. Conversely, persons on the spectrum in different categories may have a common underlying etiology. ${ }^{14}$ Therefore, the aim of the present study was to investigate the neurobiology underlying autistic features.

Inclusion criteria for the TD group included the following: 1) measured intellectual functioning within normal limits ( $\geq 85), 2$ ) normal neurologic screening, and 3) absence of any current or historical medical or psychiatric diagnoses. TD children were obtained through active recruitment. Children with any of the following were excluded from the study: 1) history of seizures, 2) focal deficits on clinical examination by a pediatric neurologist, 3) MR imaging interpreted as abnormal by a pediatric neuroradiologist, 4) dysmorphic features suggestive of a genetic syndrome, 5) history of prematurity or perinatal hypoxic-ischemic event, and 6) an inborn error of metabolism.

All children in the study were right-handed. The groups did not differ on age $(P=.25)$ or sex $(P=.27)$. Because the scans of children with ASD were clinical MR imaging studies, sedation was used as necessary by the sedation team at Children's Hospital of Michigan. None of the TD children were sedated for the MR imaging. Younger children were scanned while sleeping, and all children were monitored for movement during scan. If there was significant movement, either MR imaging was repeated or the subject was removed from the study. Written and informed consent was obtained from 1 of the parents or legal guardians of the TD participants. The Human Investigations Committee at Wayne State University granted permission for the retrieval and analysis of the clinical data and MR imaging scans of ASD children.

\section{DTI Acquisition}

All DTI scans were performed on a 3T Signa scanner (GE Healthcare, Milwaukee, Wisconsin) equipped with an 8-channel head coil at $\mathrm{TR}=1250 \mathrm{~ms}$, $\mathrm{TI}=88.7 \mathrm{~ms}, \mathrm{FOV}=240 \mathrm{~cm}, 128 \times 128$ acquisition matrix (nominal resolution $=1.89 \mathrm{~mm}$ ), contiguous $3 \mathrm{~mm}$ thickness to cover entire axial sections of whole brain by using 6 isotropic gradient directions with $b=1000 \mathrm{~s} / \mathrm{mm}^{2}, 1 b=0$ acquisition, and
NEX $=6$ to increase the signal intensity-to-noise ratio and to reduce artifacts. Approximate scanning time for the DTI acquisition was 9 minutes by using a double refocusing pulse sequence to reduce eddy current artifacts. Array spatial sensitivity encoding technique also was applied to further reduce geometric distortion due to the sequence design.

To secure uniform quality of DTI data across the subjects, the $b=$ $1000-\mathrm{s} / \mathrm{mm}^{2}$ images were corrected for eddy current distortion and any potential movements during the DTI acquisition. We used the FMRIB Software Library module for this correction (The Oxford Centre for Functional Magnetic Resonance Imaging of the Brain Software Library, http://www.fmrib.ox.ac.uk/fsl/fdt). The correction relies on a 12-parameter affine transformation.

\section{DTI Analysis}

Spatial Normalization of DTI Parameter Maps and WholeBrain Tractography. The block diagram in Fig 1 illustrates the overall steps to normalize DTI parameters across subjects in MNI space. To estimate nonlinear deformation field between native space $(x, y, z)$ and standard MNI space ( $\mathrm{u}, \mathrm{v}, \mathrm{w})$, the GM and white matter maps of individual subjects were spatially normalized to those of the TD group by using the SPM DARTEL approach. ${ }^{15}$ That is, for a given $i$ th subject, tissue probability maps (ie, GM and white matter) were first segmented from the B0 image by using the SPM segmentation module. Then, each tissue map was iteratively aligned and deformed to the corresponding map of the TD group, resulting in a nonlinear deformation field to register the voxels of native space to those of the TD population space. A composite of this nonlinear deformation field and an affine transformation between the GM coordinates of the TD space and those of the pediatric MNI space ${ }^{16}$ was used as a final deformation field, $\mathrm{M}_{\mathrm{i}}(\mathrm{x}, \mathrm{y}, \mathrm{z})$, which was defined as point-to-point mapping between the coordinates of native space and those of MNI space.

Spatial maps of diffusion tensor at every voxel of native space $(x, y$, z) were evaluated from DTI data by using an in-house implemented software incorporating a signal intensity-to-noise-ratio-weighed multivariate least square fitting approach. ${ }^{17}$ Based on the first eigen vector, $\mathrm{v}_{1}$, and 3 eigenvalues $\left(\lambda_{1}, \lambda_{2}, \lambda_{3}\right)$ of the diffusion tensor matrix, diffusion parameter maps, including FA $=\left[3 \times\right.$ variance of $\left(\lambda_{1}, \lambda_{2}, \lambda_{3}\right) /$ $\left.\left(\lambda_{1}{ }^{2}+\lambda_{2}{ }^{2}+\lambda_{3}{ }^{2}\right)\right]^{1 / 2}, \mathrm{AD}=\lambda_{1}$, and $\mathrm{RD}=\left(\lambda_{2}+\lambda_{3}\right) / 2$, were reconstructed at every voxel of native space. ${ }^{18}$ Whole-brain tractography was conducted per subject of each group by using conventional streamline tractography based on Runge-Kutta order 2 interpolation at FA $>0.20$, angular deflection $<60^{\circ}$, and step size $=0.2 \mathrm{~mm}$.

The deformation field $\mathrm{M}_{\mathrm{i}}(\mathrm{x}, \mathrm{y}, \mathrm{z})$ of individual subjects was then applied for the spatial normalization of all DTI parameters, including $\mathrm{FA}, \mathrm{AD}, \mathrm{RD}$, and whole-brain tractography [ie, the $(\mathrm{x}, \mathrm{y}, \mathrm{z})$ coordinates of individual parameters in native space can be transferred to the corresponding $(\mathrm{u}, \mathrm{v}, \mathrm{w})$ coordinates in MNI space via the $\mathrm{M}_{\mathrm{i}}(\mathrm{x}, \mathrm{y}$, $z)$ ]. We used the Frenet equation to measure the scale-free fiber curvature for normalized tracts. ${ }^{19}$ For a given fiber $\mathrm{x}(\mathrm{s})$, the curvature was measured by absolute magnitude of the first derivative of tangent vector, $\mathrm{T}(\mathrm{s})$, at spatial coordinate, $\mathrm{s}$ :

$$
\operatorname{curvature}(s)=\left|\frac{\mathrm{d} T}{\mathrm{~d} s}\right|
$$

where $\mathrm{T}(\mathrm{s})$ is defined by a gradient of $\mathrm{x}(\mathrm{s})$ (ie, $\mathrm{dx} / \mathrm{ds}$ ).

Atlas-Based Tract Filtering. To isolate the frontal lobe tracts of interest, the normalized whole-brain tracts of individual subjects were systematically sorted by using atlas-based ROIs defined in Anatomical Automatic Labeling map. ${ }^{20}$ A pair of ROIs was selected to sort 


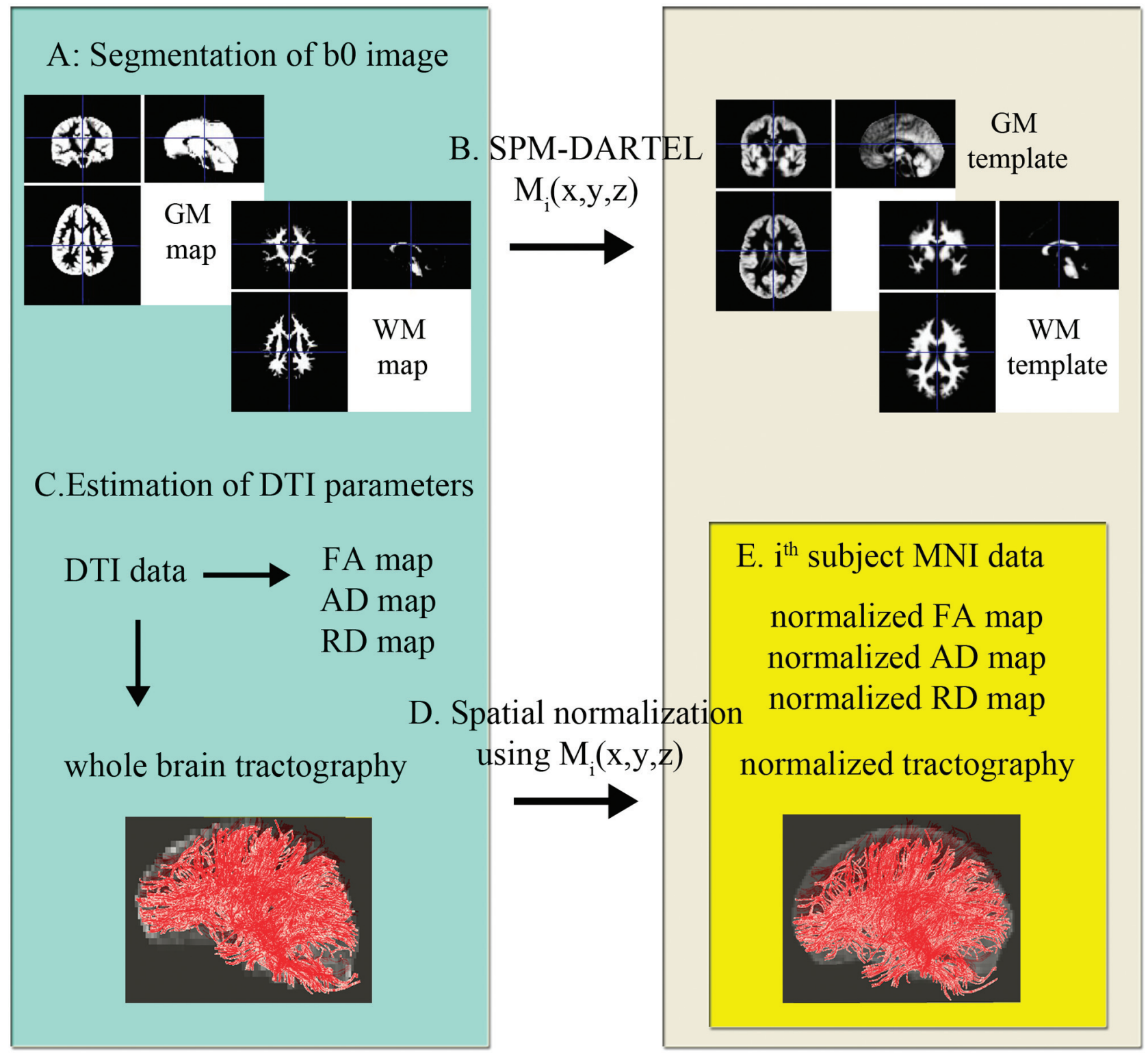

Fig 1. Steps involved in normalizing diffusion tensor data and whole-brain fiber tracts: Left and right blocks indicate the subject's native space ( $x, y, z)$ and the MNI space ( $u, v, w)$ respectively. $A$, Initially each subject's BO image was segmented into the probability maps of GM and white matter in native space. $B$, Resulting tissue maps were normalized onto corresponding pediatric probability templates in the MNI space via the SPM DARTEL deformation field, Mi(x, y, z). C, Diffusion tensor calculation and whole brain white matter tractography were performed in each subject. $D$, Resulting FA, $\mathrm{AD}$, and RD maps and the tractography results (whole-brain fiber tracts) were transferred to the MNI space by using a deformation field generated during the initial step [via point-to-point mapping of $\mathrm{Mi}(x, y, z)]$. E, Normalized tractography was used to isolate individual fiber tracts by using "atlas-based tract filtering." Finally, the isolated fiber tracts and normalized diffusivity maps were subjected to TBM analyses.

the fibers in each tract (ie, inferior frontal operculum and middle temporal region for AF, middle orbitofrontal region, and superior temporal pole for UF, and left superior orbitofrontal and right superior orbitofrontal regions for gCC). The sorted fibers of individual subjects were then pooled to form a group bundle. To ensure the consistency of individual fibers in the group pool, a group consistency map was computed by summing up the number of fibers penetrating individual voxels. This map approximates local fiber attenuation at every voxel. The voxels in which the fibers passed through in at least half of the subjects of each group were assumed to represent a "consistent pathway." The fibers existing inside the consistent pathway were subsequently considered for the group bundle that is used as an input to the subsequent TBM analysis.

TBM Analysis. The TBM analysis is a coordinate-wise statistical analysis to compare DTI parameters of multiple subjects at a common coordinate system of a specific fiber bundle. ${ }^{9}$ Initially, the most representative fiber from a given fiber bundle is selected as "prototype fiber." Uniformly sampled points on the prototype define the common coordinates, s. Independent statistical tests are performed at these common coordinates.

Here, the longest fiber penetrating the highest fiber attenuation region of a given fiber bundle in the TD group was selected as a 

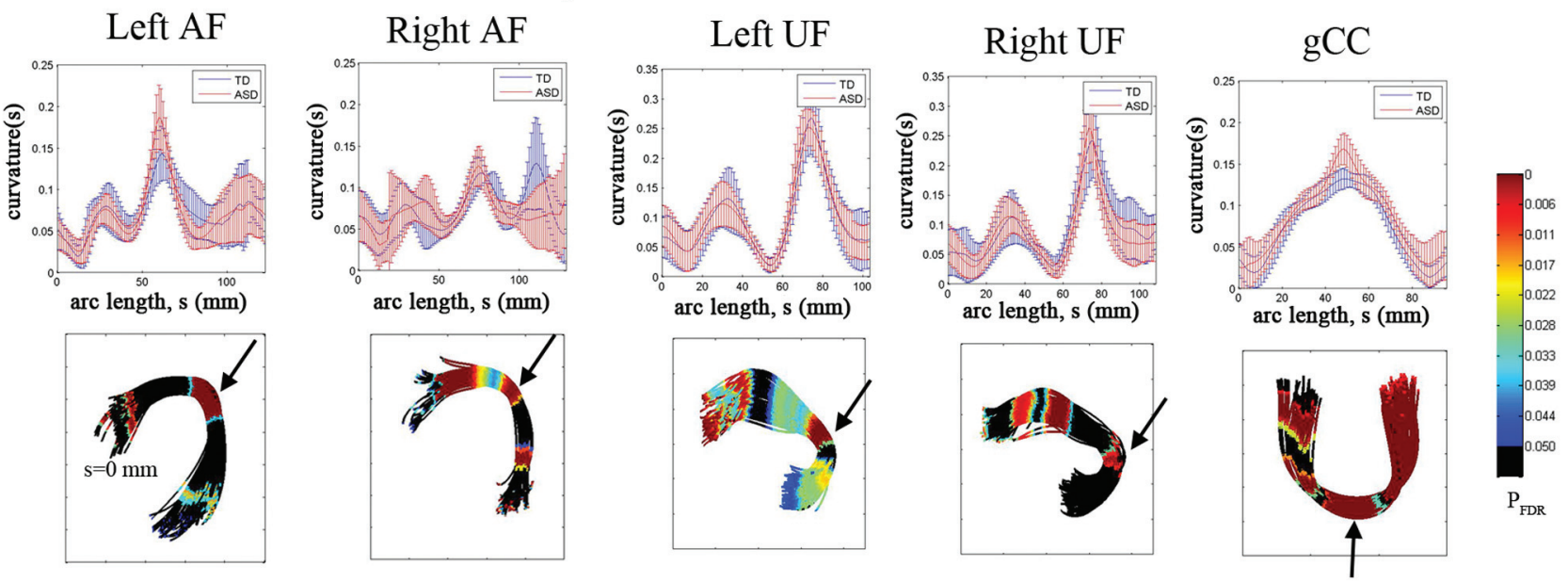

Fig 2. Fiber tract curvature estimates from TBM analysis for AF, UF, and gCC. Top panel: group mean and SD of fiber curvature at each common arc-length coordinate are plotted at the respective common coordinates. Bottom panel: for the comparison TD curvature is less than in the ASD group, the multiple-comparison $P_{F D R}$ value for the group difference in fiber curvature at each common arc-length coordinate is overlaid on a respective TD group fiber bundle. The regions with the highest $P$ values are marked with an arrow. Note that we define the first common arc-length coordinate $(s=0)$ at the anterior region for both AF and UF. The first common arc-length coordinate of the gCC is defined in the right hemisphere, which is the left side of the tract display.

\begin{tabular}{|c|c|c|c|c|c|c|}
\hline \multirow[b]{2}{*}{ Fiber Pathway } & \multicolumn{3}{|c|}{ MNI Coordinates (mm) } & \multirow[b]{2}{*}{ Group } & \multirow[b]{2}{*}{ Mean (SD) } & \multirow[b]{2}{*}{$\mathrm{P}_{\mathrm{FDR}}$} \\
\hline & $x$ & $\mathrm{y}$ & z & & & \\
\hline \multirow[t]{2}{*}{$\overline{\text { AF-left }}$} & -36.21 & -42.50 & 24.71 & TD & $0.1391(0.0313)$ & $<.001$ \\
\hline & & & & ASD & $0.1812(0.0355)$ & \\
\hline \multirow[t]{2}{*}{ AF-right } & 35.99 & -42.86 & 26.65 & TD & $0.1037(0.0196)$ & .0136 \\
\hline & & & & ASD & $0.1227(0.0226)$ & \\
\hline \multirow[t]{2}{*}{ UF-left } & -34.80 & -0.0586 & -11.48 & TD & $0.1591(0.0480)$ & .005 \\
\hline & & & & ASD & $0.1836(0.0577)$ & \\
\hline \multirow[t]{2}{*}{ UF-right } & 35.82 & 1.48 & -17.05 & TD & $0.2252(0.0683)$ & .03 \\
\hline & & & & ASD & $0.2559(0.0615)$ & \\
\hline \multirow[t]{2}{*}{ gCC } & 0.01 & 26.24 & 4.22 & TD & $0.1317(0.0126)$ & $<.001$ \\
\hline & & & & ASD & $0.1673(0.0183)$ & \\
\hline
\end{tabular}

prototype for that particular bundle and was used to define the common arc-length coordinates for that bundle. Common coordinates were placed every 2-mm arc-length of the prototype fiber (ie, 2-mm arc-length approximates single voxel size, $\approx 1.89 \mathrm{~mm}$ ). These coordinates were used as "reference points" to register the corresponding coordinates of individual fibers of the respective tracts in each subject by using the Hungarian matching algorithm. ${ }^{9,21}$ Subsequently, the DTI parameters (curvature, FA, AD, and $\mathrm{RD}$ ) of each fiber tract at each coordinate were evaluated in each subject, and group differences were examined by 2-sample $t$ test. We assessed $\mathrm{P}_{\mathrm{FDR}}$ for multiple comparisons. $^{22}$

\section{Results}

\section{Curvature TBM Analysis}

The TBM group comparisons of curvature are presented in Fig 2. For each common arc-length coordinate of individual fiber bundles, the curvature values of all registered fibers were evaluated by using equation 1 and compared across the groups. The group mean and SD are presented in the plot (blue for TD and red for ASD). The coordinates showing significant group difference $\left(\mathrm{H}_{1}\right.$ [curvature]: $\mathrm{TD}<\mathrm{ASD}$ at $\left.\mathrm{P}_{\mathrm{FDR}}<.05\right)$ are appropriately color-coded at every $2-\mathrm{mm}$ common arc-length coordinate for the entire pathway. The TBM analysis identi- fied focal coordinates showing higher curvature in the ASD group. As indicated by black arrows in Fig 2, the bending regions of individual pathways showed higher curvature. That is, the curvature is apparently increased at the parietotemporal junction for bilateral AF (left, ASD versus TD $=0.18 \pm 0.03$ versus $0.14 \pm 0.03, \mathrm{P}_{\mathrm{FDR}}<.001$; right, $\mathrm{ASD}$ versus $\mathrm{TD}=$ $0.12 \pm 0.02$ versus $\left.0.10 \pm 0.02, \mathrm{P}_{\mathrm{FDR}}=.01\right)$, at the frontotemporal junction for bilateral UF (left, ASD versus TD $=0.18 \pm$ 0.05 versus $0.16 \pm 0.04, \mathrm{P}_{\mathrm{FDR}}=.005$; right, $\mathrm{ASD}$ versus $\mathrm{TD}=$ $0.26 \pm 0.06$ versus $\left.0.22 \pm 0.06, \mathrm{P}_{\mathrm{FDR}}=.03\right)$, and at the midline of the $\mathrm{gCC}$ (ASD versus $\mathrm{TD}=0.17 \pm 0.02$ versus $0.13 \pm 0.01$, $\left.\mathrm{P}_{\mathrm{FDR}}<.0001\right)$. Table 1 summarizes the locations of junctions in MNI space, showing higher curvature in the children with ASD.

\section{Diffusivity TBM Analysis}

The results of the diffusivity analysis are presented in Fig 3. The FA was significantly reduced in children with ASD at 2 distinct focal regions, inferior frontal and middle temporal regions, of bilateral $\mathrm{AF}\left(\mathrm{P}_{\mathrm{FDR}}<.04\right)$. Similarly, the anterior and posterior ends of left UF showed significant group differences in FA (decreased in children with ASD, $\mathrm{P}_{\mathrm{FDR}}=.041$ ). The ASD group also exhibited significantly reduced FA in the 

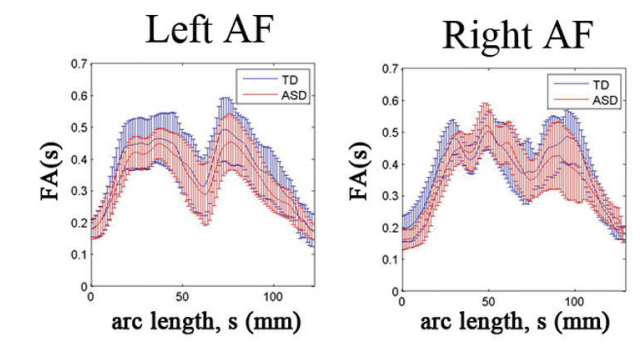

$\mathrm{H}_{0}(\mathrm{FA}): \mathrm{TD}>\mathrm{ASD}$
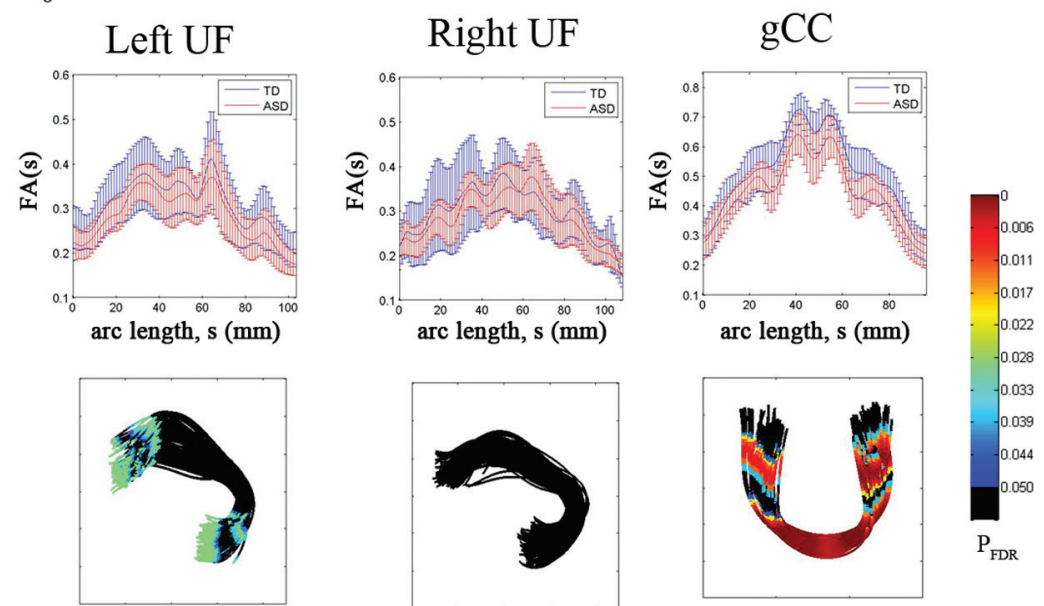

Fig 3. Fiber tract FA estimates from TBM analysis for AF, UF, and gCC. Top panel: group mean and SD of FA at each common arc-length coordinate are plotted at the respective common coordinates. Bottom panel: for the comparison of the TD group FA is greater than in the ASD group, the multiple-comparison $P_{F D R}$ value for the group difference in fiber curvature at each common arc-length coordinate is overlaid on a respective TD group fiber bundle. Note that we define the first common arc-length coordinate ( $\mathrm{s}=0$ ) at the anterior region for both $\mathrm{AF}$ and UF. The first common arc-length coordinate of the gCC is defined in the right hemisphere, which is the left side of the tract display.
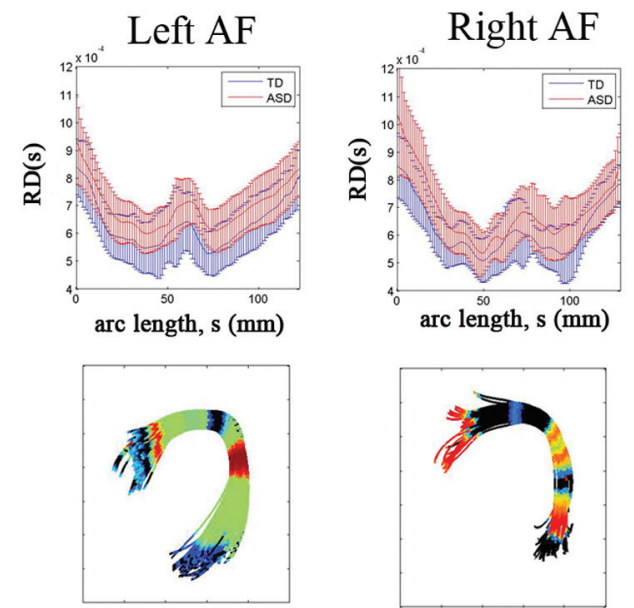

$$
\mathrm{H}_{1}(\mathrm{RD}): \mathrm{TD}<\mathrm{ASD}
$$
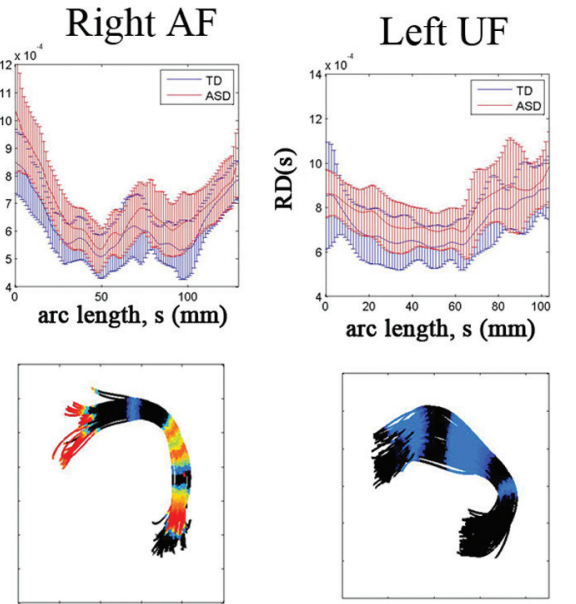
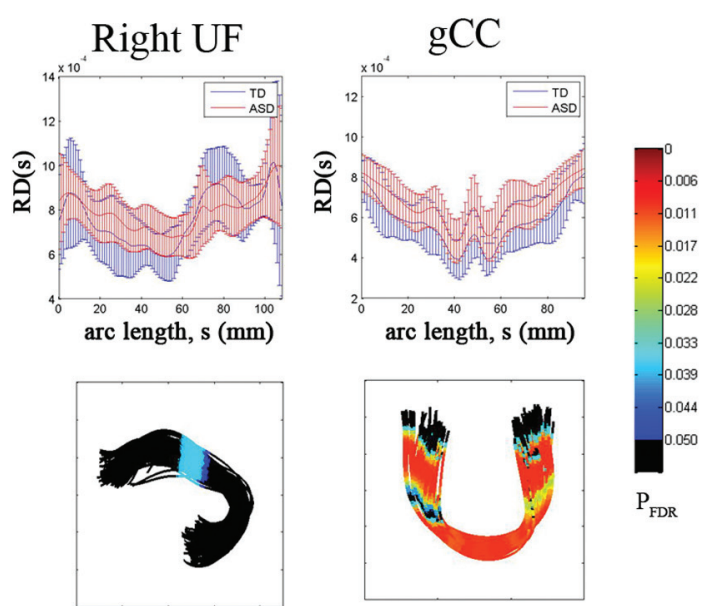

Fig 4. Fiber tract radial diffusivity estimates from TBM analysis for AF, UF, and gCC. Top panel: group mean and SD of RD at each common arc-length coordinate are plotted at the respective common coordinates. Bottom panel: for the comparison of the TD group RD is less than in the ASD group, the multiple-comparison $\mathrm{P}_{\mathrm{FDR}}$ value for the group difference in fiber curvature at each common arc-length coordinate is overlaid on a respective TD group fiber bundle. Note that we define the first common arc-length coordinate ( $\mathrm{s}=0$ ) at the anterior region for both AF and UF. The first common arc-length coordinate of the gCC is defined in the right hemisphere, which is the left side of the tract display.

central portion of gCC $\left(\mathrm{P}_{\mathrm{FDR}}=.0005\right)$. The TBM analysis of $\mathrm{RD}$ is presented in Fig 4. The children with ASD showed higher RD at most arc-length coordinates of individual pathways, especially in bilateral AF $\left(\mathrm{P}_{\mathrm{FDR}}<.025\right)$, left UF $\left(\mathrm{P}_{\mathrm{FDR}}<\right.$ $.04)$, and gCC $\left(\mathrm{P}_{\mathrm{FDR}}<.015\right)$. However, no group difference was found in $\mathrm{AD}$ for any of the tracts.

\section{Relationship between Curvature and Diffusivity}

Interestingly, we found high correlations between the values of curvature and diffusivity parameters (FA and RD) that were sampled from the same bending regions of bilateral AF, UF, and gCC of both groups. There was significant negative correlation between curvature and FA (increased curvature and decreased FA) and positive correlation between curvature and $\mathrm{RD}$ (increased curvature and increased RD) at bilateral AF,
UF, and gCC (Table 2). Compared with the TD group, the ASD group showed stronger correlations between curvature and RD. No significant correlation was found between curvature and $\mathrm{AD}$ in both groups.

\section{Discussion}

The major finding of the present study is that children in the ASD group show higher curvature and sharper bending in bilateral AF and UF, and gCC. At sharply bending regions of these pathways, the change of the curvature was positively correlated with RD but negatively correlated with FA. Our previous study in this patient population ${ }^{6}$ showed changes of the fiber tracts by quantifying "fiber length" and "fiber length profile." In the current study, further details about changes in the fiber geometry by quantifying "fiber curvature" along the 


\begin{tabular}{|c|c|c|c|c|c|c|c|c|}
\hline \multirow{3}{*}{$\begin{array}{l}\text { Fiber } \\
\text { Pathway }\end{array}$} & \multicolumn{4}{|c|}{ Curvature vs. FA } & \multicolumn{4}{|c|}{ Curvature vs. RD } \\
\hline & \multicolumn{2}{|c|}{ TD } & \multicolumn{2}{|c|}{ ASD } & \multicolumn{2}{|c|}{ TD } & \multicolumn{2}{|c|}{ ASD } \\
\hline & $R^{2}$ & $P$ & $R^{2}$ & $P$ & $R^{2}$ & $P$ & $R^{2}$ & $P$ \\
\hline Bilateral AF & 0.42 & .0011 & 0.84 & $<.0001$ & 0.00 & .7483 & 0.57 & $<.0001$ \\
\hline Bilateral UF & 0.49 & .0003 & 0.22 & .0259 & 0.00 & .9845 & 0.19 & .0433 \\
\hline gCC & 0.83 & .0001 & 0.94 & $<.0001$ & 0.61 & .0044 & 0.94 & $<.0001$ \\
\hline
\end{tabular}

Note:- $P$ value indicates the probability of getting a correlation as large as the observed value by random chance and was estimated from testing the hypothesis of no correlation at $95 \%$ confidence level.

whole path of the fiber were determined. In addition, whereas diffusion parameters were calculated and reported for the entire fiber tract in the previous study, the present study calculated the curvature-based changes in diffusion parameters. For this analysis, we used TBM. The registration is a key factor in determining the accuracy of the TBM analysis. Although the accuracy of the SPM DARTEL model has been validated in an ROI-based VBM study, ${ }^{23}$ the perfect coregistration of all fiber bundles of the brain (small and large) is difficult to achieve. Whereas reasonable coregistration can be achieved for large fiber tracts, coregistration is not very reliable for smaller fiber tracts. Therefore, although this limitation did not affect our present findings, because only large fiber tracts were analyzed in the present study, this may limit the applicability of the current method to the analysis of major fiber bundles only. In addition, we observed that the curvature is highly sensitive to 2 experimental parameters: the signal intensity-to-noise ratio of MR acquisition and the size of arc-length in the TBM analysis. The optimization of these parameters requires a larger sample size.

Several studies have reported abnormal changes of diffusivity parameters averaged over the entire fiber in the AF of children with ASD. For example, our previous study ${ }^{6}$ showed that children with ASD had significantly lower FA and higher $\mathrm{MD}$ in right $\mathrm{AF}$. Fletcher et $\mathrm{al}^{24}$ reported lower $\mathrm{FA}$ and higher $\mathrm{MD}$ in bilateral AF but less left lateralized MD and FA in highfunctioning adolescents with ASD. These findings are also consistent with our present results showing reduced FA and increased RD in bilateral AF fibers in children with ASD.

Primarily, the UF contains fibers connecting amygdala and orbitofrontal cortex (amygdalo-orbitofrontal circuit). Thus, the UF plays a critical integrative role in social cognitive and emotional functioning. The present study demonstrated that children with ASD showed aberrant changes in diffusivity and geometry in bilateral UF; these changes may be related to impairment in amygdalo-orbitofrontal circuit function in children with ASD. ${ }^{25,26}$

The reduced FA in gCC of the children with ASD has been reported by several studies. ${ }^{3,27,28}$ We also found lower FA and higher RD in gCC of the children with ASD. Especially, our TBM analysis revealed that these changes are more predominant in the midline of the $\mathrm{gCC}$, which is the segment with high curvature. Although one previous study reported no significant difference in overall CC shape and contour in children with ASD associated with macrocephaly, ${ }^{29}$ it approximated the shape of the CC by measuring only 5 angulations between 4 lines enclosing the entire CC in T1-weighted midsagittal images. This approach would not be sensitive enough to reveal fine structural differences that the TBM analysis could detect at voxel resolution level.

\section{Microstructural Basis of DTI Abnormalities in Autism}

Higher curvature, higher RD, and lower FA in children with ASD are consistent with increased number of fibers ${ }^{5,6}$ and may be related to higher attenuation of cortical minicolumns demonstrated in previous studies. ${ }^{30,31}$ In "minicolumnopathy" of ASD, the cortical minicolumns contain numerous, smaller neurons that are densely packed. Thus, it could be anticipated that thinner and more numerous axons would emanate from these minicolumns. The interpretation of more numerous, thinner axons is also consistent with our previous studies of increased number of fibers in frontal lobe tracts. ${ }^{5,6}$ Thinner axons will disproportionately decrease the axon volume and increase interaxonal volume. The overall effect is to decrease the diffusion barriers to axons that can result in increased $\mathrm{RD}$ and decreased FA. Thus, the profile of abnormalities (higher curvature, higher $\mathrm{RD}$, and lower FA) is consistent with the microstructural abnormalities of thinner and more numerous axons.

\section{Aberrant Properties of Frontal Lobe Fibers in ASD}

In this study, the children with ASD showed lower FA confined to the inferior frontal and middle temporal regions of bilateral AF, with higher $\mathrm{RD}$ at the same regions. This set of finding implies 2 possible scenarios: 1) existence of thin axons with high attenuation as discussed in the previous section and 2) decreased organization of fibers resulting in more tortuosity at local areas. Of these 2 possibilities, higher curvature at flexion points of a fiber tract would be more consistent with the presence of thin axons with high attenuation. Thinner fibers would be expected to lead to slower conduction velocity and may be related to reports of delayed auditory evoked potentials in ASD. ${ }^{32}$ Thinner and more numerous axons in AF might degrade language functionality and be interpreted as the underconnectivity previously demonstrated in ASD. ${ }^{33}$

\section{Conclusions}

The present study found higher curvature and significant curvature-dependent diffusivity changes in bilateral AF, UF, and gCC in children with ASD. These changes are likely to be due to abnormally developed axons of these frontal lobe pathways, which provide new insight into ASD specific white matter malformation characterized by higher attenuation of thinner axons in frontal lobe tracts.

Disclosures: Diane S. Chugani, Consultant: Shire, Details: Autism workshop speaker Dec. 13, 2010.

\section{References}

1. Barnea-Goraly $\mathrm{N}$, Kwon $\mathrm{H}$, Menon $\mathrm{V}$, et al. White matter structure in autism: preliminary evidence from diffusion tensor imaging. Biol Psychiatry 2004;55:323-26

2. Ben Bashat D, Kronfeld-Duenias V, Zachor DA, et al. Accelerated maturation 
of white matter in young children with autism: a high b value DWI study. Neuroimage 2007;37:40-47

3. Keller TA, Kana RK, Just MA. A developmental study of the structural integrity of white matter in autism. Neuroreport 2007;18:23-27

4. Lee JE, Bigler ED, Alexander AL, et al. Diffusion tensor imaging of white matter in the superior temporal gyrus and temporal stem in autism. Neurosci Lett 2007;7:127-32

5. Sundaram SK, Kumar A, Makki MI, et al. Diffusion tenor imaging of frontal lobe in autism spectrum disorder. Cereb Cortex 2008;18:2659-65

6. Kumar A, Sundaram SK, Sivaswamy L, et al. Alterations in frontal lobe tracts and corpus callosum in young children with autism spectrum disorder. Cereb Cortex 2010;20:2103-13.

7. Cheng Y, Chou KH, Fan YT, et al. Atypical development of white matter microstructure in adolescents with autism spectrum disorders. Neuroimage 2010;50:873-82

8. Mori S, Crain BJ, Chacko VP, et al. Three-dimensional tracking of axonal projections in the brain by magnetic resonance imaging. Ann Neurol 1999;45:265-69

9. O’Donnell LJ, Westin CF, Golby AJ. Tract-based morphometry for white matter group analysis. Neuroimage 2009;45:832-44

10. Smith SM, Jenkinson M, Johansen-Berg H, et al. Tract-based spatial statistics: voxelwise analysis of multi-subject diffusion data. Neuroimage 2006;31:1487-505

11. Lee JE, Chung MK, Lazar M, et al. A study of diffusion tensor imaging by tissue-specific smoothing-compensated voxel-based analysis. Neuroimage 2009;44:870-83

12. Rutter M, Bailey A, Lord C. Social Communication Questionnaire (SCQ). Los Angeles: Western Psychological Services; 2003

13. Gilliam J, Gilliam Autism Rating Scales. Austin, Texas: Pro-Ed; 1995

14. Bill BR, Geschwind DH. Genetic advances in autism: heterogeneity and convergence on shared pathways. Curr Opin Genet Dev 2009;19:271-78

15. Ashburner J. A fast diffeomorphic image registration algorithm. Neuroimage 2007;38:95-113

16. Wilke M, Schmithorst VJ, Holland SK. Assessment of spatial normalization of whole-brain magnetic resonance images in children. Hum Brain Mapp 2002;17:48-60

17. Basser PJ, Mattielo J, Le Bihan D. MR diffusion tensor spectrography and imaging. Biophys J 1994;66:259-67

18. Basser PJ, Pierpaoli C. Microstructural and physiological features of tissues elucidated by quantitative-diffusion-tensor MRI. JMR B 1996;111:209-19
19. Batchelor PG, Calamante F, Tournier JD, et al. Quantification of the shape of fiber tracts. Magn Res Med 2006;55:894-903

20. Tzourio-Mazoyer N, Landeau B, Papathanassiou D, et al. Automated anatomical labelling of activation in SPM using a macroscopic anatomical parcellation of the Montreal Neurological Institute MRI single-subject brain. Neuroimage 2002;15:273-89

21. Kuhn HW. The Hungarian method for the assignment problem. Nav Res Logist Q 1955;2: 83-91

22. Yekutieli D, Benjamini Y. A resampling based false discovery rate controlling multiple test procedure. J Stat Plan Inference 1999;82:171-96

23. Bergouignan L, Chupin M, Czechowska Y, et al. Can voxel based morphometry, manual segmentation and automated segmentation equally detect hippocampal volume differences in acute depression. Neuroimage 2009:45: 29-37

24. Fletcher PT, Whitaker RT, Tao R, et al. Microstructural connectivity of the arcuate fasciculus in adolescents with high-functioning autism. Neuroimage 2010;51:1117-25

25. Bachevalier J, Loveland KA. The orbitofrontal-amygdala circuit and self-regulation of social-emotional behavior in autism. Neurosci Biobehav Rev 2006;30:91-117

26. Dawson G, Meltzoff AN, Osterling J, et al. Neuropsychological correlates of early symptoms of autism. Child Dev 1998;69:1276-85

27. Alexander AL, Lee JE, Lazar M, et al. Diffusion tensor imaging of the corpus callosum in autism. Neuroimage 2007;34:61-73

28. Ding XQ, Sun Y, Kruse B, et al. Microstructural callosal abnormalities in normal-appearing brain of children with developmental delay detected with diffusion tensor imaging. Eur Radiol 2009;19:1537-43

29. Rice SA, Bigler ED, Cleavinger HB, et al. Macrocephaly, corpus callosum morphology, and autism. J Child Neurol 2005;20:34-41

30. Casanova MF, Buxhoeveden DP, Switala AE, et al. Minicolumnar pathology in autism. Neurology 2002;58:428-32

31. Casanova MF, van Kooten IA, Switala AF, et al. Minicolumnar abnormalities in autism. Acta Neuropathol 2006;112:287-303

32. Roberts TP, Khan SY, Rey M, et al. MEG detection of delayed auditory evoked responses in autism spectrum disorders: towards an imaging biomarker for autism. Autism Res 2010;3:8-18

33. Just MA, Cherkassky VL, Keller TA, et al. Cortical activation and synchronization during sentence comprehension in high-functioning autism: evidence of underconnectivity. Brain 2004;127:1811-21 